
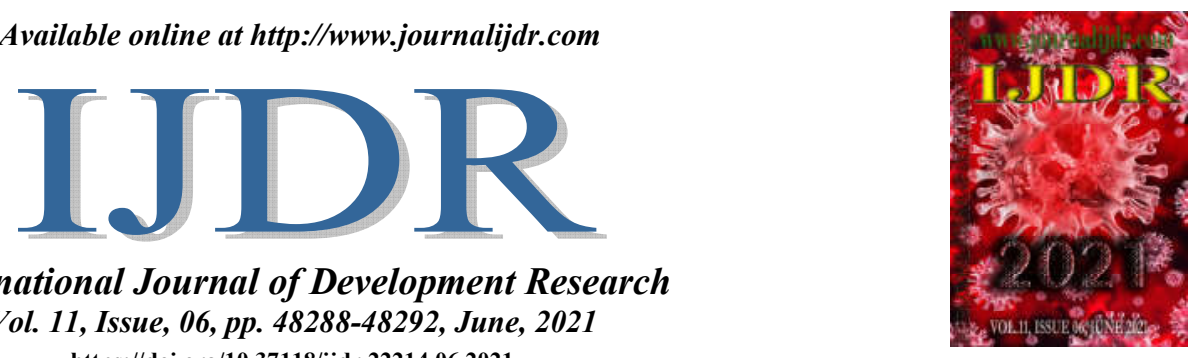

ISSN: 2230-9926

International Journal of Development Research

Vol. 11, Issue, 06, pp. 48288-48292, June, 2021

https://doi.org/10.37118/ijdr.22214.06.2021

\title{
CONHECIMENTO DE ENFERMEIROS INTENSIVISTAS ACERCA DO DIAGNÓSTICO DEENFERMAGEM RISCO DE OLHO SECO
}

1Karymi Saraty Flor Mesquita, ${ }^{2}$ Débora do Socorro Silva de Souza, ${ }^{3}$ Tassio Ricardo Martins da Costa, ${ }^{4}$ Widson Davi Vaz de Matos, ${ }^{5}$ Daniele Ferreira Bezerra, ${ }^{6}$ Gleyce Kelly Sousa Ferreira, ${ }^{7}$ Carla de Nazaré Benjamin da Silva Marques, ${ }^{8}$ Adilson Mendes de Figueiredo Júnior, ${ }^{9}$ Yasmin Martins de Sousa, ${ }^{10}$ Tania de Sousa Pinheiro Medeiros, ${ }^{11}$ Marta Cleonice Cordeiro de Assunção, ${ }^{12}$ Daniele Lima dos Anjos Reis, ${ }^{13}$ Milena Coelho Fernandes Caldato, ${ }^{14}$ Renata Campos de Sousa Borges, ${ }^{15}$ Samuel Oliveira Vera, ${ }^{16}$ Tatheanne da Silva Trindade dos Santos, ${ }^{17}$ Barbara Alves Ruela de Azevedo Ruivo, ${ }^{18}$ Karen Silva de Castro, ${ }^{19}$ Emilly Barbara Pereira da Fonseca, ${ }^{20}$ Diélig Teixeira, ${ }^{21}$ Marlene Simões e Silva, ${ }^{22}$ Odaléa Larissa dos Santos, ${ }^{23}$ Diego João de Lima Arrais, ${ }^{24}$ Priscila Morgana da Silva Caldeira, ${ }^{25}$ Fabiany Bezerra Barbosa, ${ }^{26}$ Elias Costa Monteiro, ${ }^{27}$ Miquéias Bailão Teixeira and ${ }^{28}$ Maicon de Araujo Nogueira

1Enfermeira, Universidade da Amazônia (UNAMA). Belém, Pará, Brasil; 2Enfermeira, Universidade da Amazônia (UNAMA). Belém, Pará, Brasil; Enfermeiro. Mestrado em andamento, Universidade do Estado do Pará (UEPA).

Editor-chefe, Editora Neurus. Belém, Pará, Brasil; ${ }^{4}$ Enfermeiro. Mestrado em andamento, Universidade do

Estado do Pará (UEPA). Especialista em Oncologia, modalidade residência multiprofissional. Belém, Pará, Brasil;

${ }^{5}$ Acadêmica de Enfermagem. Universidade da Amazônia (UNAMA). Belém, Pará, Brasil; ${ }^{6}$ Acadêmica de

Enfermagem. Belém, Pará, Brasil; ${ }^{7}$ Enfermeira. Especialista em Enfermagem em terapia intensiva adulto. Universidade Federal do Pará (UFPA). Belém, Pará, Brasil; ${ }^{8}$ Enfermeiro. Doutorado em andamento em Saúde

Pública, Universidade Federal do Ceará (UFC). Mestre em Enfermagem, Universidade do Estado do Pará (UEPA).

Especialista em Enfermagem do Trabalho, Centro Universitário Metropolitano da Amazônia (UNIFAMAZ).

Belém, Pará, Brasil; ${ }^{9}$ Enfermeira. Mestre em Enfermagem, Universidade Federal do Pará (UFPA). Especialista em Terapia Intensiva, Associação de Medicina Intensiva Brasileira (AMIB), Especialista em Enfermagem em Clínica Cirúrgica, modalidade residência multiprofissional. Belém, Pará, Brasil; ${ }^{10}$ Enfermeira. Mestrado em andamento, Universidade do Estado do Pará (UEPA). Especialista em Enfermagem ginecológica e obstétrica.

Tucuruí, Pará, Brasil; ${ }^{11}$ Enfermeira. Mestrado em andamento, Universidade do Estado do Pará (UEPA).

Especialista em Enfermagem em Nefrologia e Urologia, Escola Superior da Amazônia (ESAMAZ). Atua no Departamento de Vigilância Epidemiológica (DEVS) - Secretaria de Vigilância em Saúde (SESMA). Belém, Pará, Brasil; ${ }^{12}$ Enfermeira, Mestre em Ensino em Saúde na Amazônia (ESA), Universidade do Estado do Pará (UEPA).

Especialista em Enfermagem Oncológica. Docente daUniversidade do Estado do Pará (UEPA) e da Faculdade

Gamaliel, em Tucuruí/PA. Enfermeira do Hospital Regional de Tucuruí. Pará, Brasil; ${ }^{13}$ Médica. Docente do Programa de Mestrado Profissional Ensino em Saúde na Amazônia (ESA), Universidadedo Estado do Pará

(UEPA), Belém, Pará, Brasil; ${ }^{14}$ Doutoranda no Programa de Doutorado Profissional Ensino em Saúde na Amazônia (ESA), Universidade do Estado do Pará (UEPA). Tucuruí, Pará, Brasil; 15Enfermeiro. Associação de

Ensino Superior do Piauí (AESPI). Especialista em Enfermagem Obstétrica. Especialista em Enfermagem Oncológica.Mestre em Epidemiologia e Vigilância em Saúde. Instituto Evandro Chagas. Belém, Pará, Brasil;

16Enfermeira. Mestrado em andamento em Ciências da Saúde, Instituição Pequeno Príncipe. Especialista em

Ginecologia e Obstetrícia e em auditoria e Compliance em Saúde. Belém, Pará, Brasil; ${ }^{17}$ Enfermeira. Mestre em

Enfermagem. Especialista em Terapia Intensiva Adulto e Neonatal e em Saúde Coletiva. Belém, Pará, Brasil;

${ }^{18}$ Acadêmica de Enfermagem. Universidade do Estado doPará (UEPA). Pará, Brasil; ${ }^{19}$ Enfermeiro. Universidade da Amazônia (UNAMA). Belém, Pará, Brasil; ${ }^{20}$ Enfermeiro. Especialista em Terapia Intensiva Adulto e Neonatal e em Oncologia. Pará, Brasil; ${ }^{21}$ Enfermeira. Universidade José do Rosário Vellano. Especialista em terapia intensiva. Pará, Brasil; ${ }^{22}$ Enfermeira. Universidade daAmazonia (UNAMA). Coordenadora de vigilância epidemiológica em saúde. Curuça, Pará, Brasil; ${ }^{23}$ Enfermeiro. Mestrado em andamento em Enfermagem, Universidade Federal do Pará (UFPA), Belém, Pará, Brasil; ${ }^{24}$ Enfermeira, Universidade Federal do Maranhão. 
Especialista em Ginecologia e Obstetrícia e em Gestão em Saúde Pública com ênfase em saúde coletiva e da família. Belém, Pará, Brasil; ${ }^{25}$ Acadêmica de Enfermagem. Faculdade Gamaliel- FATEFIG. Tucuruí, Pará, Brasil;

${ }^{26}$ Acadêmico de Enfermagem. Faculdade Pan Amazônica (FAPAN). Belém, Pará, Brasil; ${ }^{27}$ Enfermeiro.

Universidade da Amazonia (UNAMA). Belém, Pará, Brasil; ${ }^{28}$ Enfermeiro. Mestre em Ensino em Saúde na

Amazônia, Universidade do Estado doPará (UEPA). Doutorando, Programa de Doutorado Profissional em Ensino em Saúde naAmazônia. Universidade do Estado do Pará (UEPA). Belém, Pará, Brasil.

\section{ARTICLE INFO}

\section{Article History:}

Received $08^{\text {th }}$ March, 2021

Received in revised form

$26^{\text {th }}$ April, 2021

Accepted $11^{\text {th }}$ May, 2021

Published online $30^{\text {th }}$ June, 2021

\section{Key Words:}

Terapia Intensiva; Ceratite; Úlcera na córnea;

Cuidados de Enfermagem.

*Corresponding author:

Luiz Antonio Soares Cardoso

\begin{abstract}
Objetivo:analisar o conhecimento de enfermeiros acerca do diagnóstico de Enfermagem Risco de Olho Seco em uma Unidade de Terapia Intensiva. Método: estudo descritivo com abordagem qualitativa,desenvolvido com onze enfermeiros integrantes do quadro de funcionários do hospital escolhido como cenário da pesquisa. $\mathrm{O}$ estudo foi desenvolvido nas unidades de tratamento intensivo de um hospital público de média a alta complexidade, vinculado ao SUS, referência em oncologia, localizado na cidade de Belém, Estado do Pará. As informações foram analisadas pela técnica de análise de conteúdo de Bardin. Resultados: obteve-se três categorias: conhecimento dos enfermeiros acerca dos métodos de intervenç̃es utilizados para prevenção do olho seco; conhecimento dos enfermeiros acerca dos fatores de risco para o surgimento do olho seco; conhecimento dos enfermeiros acerca do diagnóstico de enfermagem risco de olho seco. Conclusão: o conhecimento dos enfermeiros acerca da temática se mostrou fragilizado, ou seja, os resultados permitem inferir que o entendimento dos enfermeiros sobre a temática é superficial, pois não houve respostas consolidadas e detalhadas a respeito do conceito de risco do olho seco, bem como os principais fatores de risco discriminados nos diagnósticos de enfermagem.
\end{abstract}

Copyright (C) 2021, Luiz Antonio Soares Cardoso et al. This is an open access article distributed under the Creative Commons Attribution License, which permits unrestricted use, distribution, and reproduction in any medium, provided the original work is properly cited.

Citation: Taynah da Silva Pinheiro, Rondinele Mendes da Silva and Selma Rodrigues de Castilho. "Conhecimento dos enfermeiros intensivistas acerca de diagnóstico deenfermagem risco de olho seco.", International Journal of Development Research, 11, (06), 48288-48292.

\section{INTRODUCTION}

Nas Unidades de Terapia Intensiva, comumente, são internados pacientes com alto grau de gravidade clínica, em sua maioria sedados, em coma, com suporte ventilatório ou em uso de diversos medicamentos e com os mecanismos de proteção ocular comprometido. No contexto do ambiente intensivo, até o momento, pouca importância tem sido atribuída ao cuidado de danos relacionados à percepção visual, uma vez que sua abordagem requer conhecimentos específicos, participação de uma equipe multiprofissional qualificada e cuidados para prevenção e redução de danos oculares (ARAÚJO et al. 2016). A unidade intensiva é marcada pela preocupação das equipes multiprofissionais quanto à estabilização dos principais sistemas vitais, como o sistema respiratório, cardiovascular e neurológico. Todavia, não há práticas satisfatóriasque garantam importância à implementação de medidas preventivas e intervenções para questões classificadas como secundárias, como o cuidado com as alterações na superfície ocular. Em continuidade, como na maioria das vezes, os pacientes são incapazes de relatar sintomas oculares, frequentemente, esse problema é colocado em segundo plano (ARAÚJO; RIBEIRO; CHIANCA, 2017). A falta de cuidados específicos pode acarretar a Síndrome do Olho Seco, também conhecida como Ceratoconjuntivite seca, ou síndrome da lágrima disfuncional, é uma afecção multifatorial causada pela produção lacrimal inadequada e/ou rápida evaporação do filme lacrimal a partir de fatores diversos, como doença inflamatória, fatores ambientais, alterações hormonais, ou idade. Nesse contexto, no que concerne os ambientes intensivos, o filme lacrimal está comprometido em virtude da desordem nos mecanismos responsáveis pela lubrificação e proteção ocular (FERNANDES et al. 2018). Esses pacientes, devido ao comprometimento de mecanismos protetores dos olhos, são suscetíveis à desidratação, abrasão, perfuração e infecção corneana, todavia, o cuidado ocular é imprescindível e pode e deve ser prescrito por um enfermeiro. A ausência desse tipo de cuidado pode impactar, de forma negativa, à vida dos pacientes após a alta da unidade intensiva, lesões e até cegueira podem ocorrer e, caso as córneas sejam tecidos em potenciais para doação, podem ser perdidas se o cuidado não for prestado adequadamente (QUEIROZ et al. 2018).
Ademais, o estudo de coorte de Werli-Alvarenga et al. (2016) realizado no Brasil com 254 pacientes submetidos aos cuidados intensivos, apontaram que as lesões no tecido corneano foram incidentes em $59,4 \%$ dos pacientes e o tempo médio de aparição da lesão foi de 8,9 dias. Outrossim, esse estudo reforça que durante o período de internação, o enfermeiro e a equipe médica devem, obrigatoriamente, realizar a avaliação corneana para que ações de prevenção sejam implementadas de forma correta e precoce.Nesse interim, considera-se que a abordagem preventiva com a implementação de cuidados oculares específicos é fundamental, pois a não prevenção do olho seco pode impactar, negativamente, na vida dos pacientes (ARAUJO; RIBEIRO; CHIANCA, 2017).

Objetivo: Analisar o conhecimento de enfermeiros acerca do diagnóstico de Enfermagem Risco de Olho Seco em uma Unidade de Terapia Intensiva.

\section{MATERIAIS E MÉTODOS}

Participantes: Participaram do estudo onze enfermeiros integrantes do quadro de funcionários do hospital escolhido como cenário da pesquisa.

Aspectos éticos: O projeto seguiu todas as recomendações e preceitos éticos da Resolução 466/2012 do Conselho Nacional de Saúde e, nesse sentido, a pesquisa foi devidamente autorizada pelo Comitê de Ética em Pesquisa, por meio do Certificado de Apresentação para Apreciação Ética (CAAE) n n 06943319.1.0000.5173, sob o número do Parecer: 3.211.770.

Tipo de estudo: Estudo descritivo com abordagem qualitativa.

\section{Procedimentos metodológicos}

Cenário do estudo: $O$ estudo foi desenvolvido nas unidades de tratamento intensivo de um hospital público de média a alta complexidade, vinculado ao SUS, referência em oncologia, localizado na cidade de Belém, Estado do Pará, no período que compreende a coleta de dados foi no mês de abril a maio de 2019. 
Fonte de dados: A coleta de dados foi realizada de forma individual, as falas foram gravadas em um dispositivo móvelpara evitar viés de memória, permitindo que o entrevistado discorresse sobre o assunto sem tempo pré-determinado para as respostas. Com a finalidade de resguardar o anonimato dos participantes, as falas foram identificadas por códigos alfa numéricos, onde "E", significa enfermeiro, seguido do número na ordem que os participantes foram entrevistados. Realizamos uma entrevista semiestruturada e como instrumento um roteiro de entrevista, com quatro perguntas elaboradas pelos pesquisadores. Após a transcrição das dos discursos e a análise dos dados,foram realizadas por meio de análise de conteúdoque procura conhecer o que está por trás das palavras, oscilando entre objetividade e a subjetividade, enfatizando a verificação das falas ou escrita, assim como a interpretação do pesquisador (Bardin, 2016).

Critérios de inclusão e de exclusão: Foram incluídos enfermeiros de ambos os gêneros, que fizessem parte do quadro de funcionários da instituição e que desenvolvessem suas atividades laborais na unidade de terapia intensiva adulta. Foram excluídos enfermeiros residentes, além de profissionais indisponíveis no momento da entrevista, por motivo de falta, férias, licença, folga, entre outros.

Coleta e organização de dados: As entrevistas foram gravadas em aparelho digital, com anuência dos participantes, a fim de garantir maior fluência, fidedignidade e agilidade ao processo, além de melhor interação entre entrevistador e entrevistado. Em seguida, os dados foram transcritos na íntegra para o programa MicrosoftOffice Word 2016, posteriormente, passaram por processo analítico e descritivo a partir do referencial da Análise de Conteúdo de Bardin.

Análise dos dados: Os dados coletados foram submetidos à técnica de análise de conteúdo, a partir disso, a análise dos dados permitiu a construção de três categorias temáticas que englobam os objetivos específicos deste estudo. Foram respeitadas as Resoluções 510/16 do Conselho Nacional de Saúde e da Comissão Nacional de Ética em Pesquisa. $\mathrm{O}$ aceite da participação foi estabelecido por meio de assinatura do Termo de Consentimento Livre e Esclarecido, em duas vias. Para garantir o sigilo e anonimato dos participantes, optamos por realizar a codificação com a letra' $E$ ' para designar os enfermeiros, seguido de numeração ordinal.

\section{RESULTADOS}

Constatamos que dentre os onze participantes, sete eram do gênero feminino e quatro do gênero masculino. A idade variou de 29 a 54 anos, e os indivíduos eram em sua maioria, casados $(60,5 \%)$. Todos os participantes, possuíam pós-graduação Lato Sensu, com tempo de serviço variável entre 1 a 25 anos de experiência. A partir disso, realizamos a caracterização dos participantes de acordo com o estado civil, gênero, idade, pós-graduação, tempo de serviço e se o local de trabalho possui estrutura adequada, conforme quadro 1. Os dados foram submetidos à técnica de análise de conteúdo proposta por Bardin, esse processo faz com que o pesquisador retorne as bases teóricas em busca de embasamento científico para fornecer sentido as interpretações (LIMA\&MANINI, 2016). Por tanto, a análise dos dados permitiu a construção de três categorias temáticas que englobam o conhecimento dos enfermeiros intensivistas sobre o risco de olho seco em pacientes sob terapia intensiva.

Conhecimento dos enfermeiros acerca do diagnóstico de enfermagem risco de olho seco

Essa categoria descreve o conhecimento dos enfermeiros sobre conceito do diagnóstico de enfermagem risco de olho seco. Os enfermeiros relacionaram diagnóstico aos pacientes que tem dificuldade para o fechamento completo das pálpebras, que estão em risco de desenvolver lesões associadas a umidificação, como se pode observar nas falas abaixo:

"[...] ainda não é um diagnóstico existente, é o risco e esse risco geralmente ele aparece quando o paciente não consegue fazer o fechamento completo das pálpebras levando a exposição desse olho $[\ldots] " .(E 1)$

Ocorre quando o paciente apresenta alguma impossibilidade de fazer o movimento de piscar o olho, já que quando a gente pisca, a gente tem o movimento de renovar a lágrima, é um lubrificante [...]”. (E2)

"Risco de olho seco é quando o paciente não consegue fechar as pálpebras e não consegue lubrificar o globo ocular [...]”. (E5).

Destacamos que os entrevistados apresentaram um déficit de conhecimento sobre o conceito de diagnóstico, ou seja, os enfermeiros não reconhecem o risco de olho secocomo uma condição em potencial para o desenvolvimento de lesões oculares em pacientes críticos, como podemos observar:

"O diagnóstico de enfermagem de risco é a possibilidade de o paciente ter alguma situação relacionado ao tratamento dele dentro do hospital. Como nós estamos falando de risco de olho seco está relacionado, no caso, com a possibilidade de o paciente apresentar complicações na esclerótica [...]”.'. (E6)

"É dificil falar, porque nessa UTI a gente já tem os diagnósticos de enfermagem pré-estabelecidos, então o conceito do diagnóstico fica dificil. Porque não sei como definir". (E7)

"[...] O risco de olho seco é um risco de haver um comprometimento da mucosa ocular, da esclerótica [...]”. (E8)

Conhecimento dos enfermeiros acerca dos fatores de risco para o surgimento do olho seco: Em continuidade, na segunda categoria, buscamos identificar se os enfermeiros conheciam os fatores de risco predisponentes para o surgimento do olho seco. Logo, observamos que esses profissionais, em sua maioria, associam olho seco apenas aos procedimentos sedativos e ao fechamento incompleto das pálpebras.

"Um fator de risco é o ambiente muito propicio ao ressecamento da mucosa ocular, por exemplo o ambiente do centro de terapia intensiva que é um ambiente refrigerado. $O$ outro fator de risco é o fechamento incompleto das pálpebras que pode ser do próprio paciente. O terceiro fator de risco é a falta de lagrimas para fazer a umidificação do globo ocular". (E1)

"É a sedação do paciente, ou seja, esse paciente já tem uma prédisposição, por ser diabético, que já tem uma pré-disposição de alguma alteração do globo ocular por causa das sequelas, e outro tipo de patologia, paciente que já vem com tipo de neoplasia do globo ocular que deve ser observado". (E2)

"Principalmente paciente em uso de sedação e em uso de ventilação, pois não é possível que esse paciente não consiga fechar completamente os olhos e em relação a iluminação também, paciente que ocorre edema também de esclerótica, então tem risco para ocorre a lesão". (E4)

Conhecimento dos enfermeiros acerca dos métodos de intervenções utilizados para prevenção do olho seco: Por conseguinte, na categoria três, foi notório a carência de protocolos que torne como rotina no setor a prevenção do olho seco. Ressaltamos que os 11 profissionais relataram a utilização de gaze umedecida com solução salina a $0,9 \%$ como método utilizado para a prevenção do olho seco, desse total, apenas um profissional relatou aplicar a Sistematização da Assistência de Enfermagemdurante os cuidados oculares e trêsrelataram fazer a avaliação contínua. A partir das respostas, observamos déficit de conhecimentos sobre os métodos de prevenção, assim como a falta de padronização de cuidados com os olhos, como observados nos discursos a seguir:

"A gente consegue fazer o fechamento completo, umidificando as gazes, colocando em cima das pálpebras pra tentar deixar essa mucosa ocular um pouco mais lubrificada[...]". (E1) 
"O método que a gente sempre utiliza é a "inspeção", e a limpeza do globo ocular, pois mantemos o globo ocular umidificado. Quando o paciente não consegue fazer o movimento de piscar, é necessário umidificar com solução fisiológica e fazer a manutenção para que essa solução não sequer, e assim evitar que fica apenas a gaze seca, pois isso pode lesar o globo ocular". (E2)

"O método utilizado que a gente comumente trabalha é a questão da umidificação das gazes e colocar em cima dos olhos". (E5)

"[...] manter uma hidratação, ou seja, através da instilação de solução fisiológica ou o colírio se for o caso, embora não seja muito comum aqui [...]”.'. (E8)

Outrossim, grande ênfase foi dada para a falta de assistência nos cuidados de enfermagem que diz respeito ao olho seco, ou seja, foi observado relatos relatando que apenas quando o paciente é um possível doador de córnea os devidos cuidados são prestados.

"[...] as vezes a gente passa batido, tu olhas todos os itens, você olha a lesão por pressão, você olha todos esses aspectos, mas a gente não olha especificamente para córnea, para o olho, então a gente precisa realmente ter mais atenção para essa questão". (E3)

"[...] comecei a ter esse cuidado com os olhos em outro no outro hospital que trabalho, pois lá existe o protocolo de cuidados para pacientes doadores de córnea [...]". (E6)

"A prevenção justamente é isso, eu avalio o paciente, na verdade a função do enfermeiro é essa, avaliar o paciente e prever riscos [...], principalmente a minha conduta é hidratar esse olho do paciente com soro fisiológico embebedado numa pétala de gaze e sempre tentando deixar umidificado, pois o paciente pode ser um possivel doador $[\ldots] "$. (E6).

\section{DISCUSSÃO}

Em relação ao conhecimento dos enfermeiros sobre conceito do diagnóstico de enfermagem risco de olho seco, os profissionais associaram o olho seco aos pacientes que tem dificuldade para o fechamento completo das pálpebras, podendo desenvolver lesões associadas ao ressecamento. Destacamos, ainda, que alguns entrevistados associaram o risco de olho seco com a questão esclerótica, e outros demonstraram um déficit de conhecimento sobre o conceito de diagnóstico, ou seja, não reconhecem esse problema como prejudicial aos olhos dos pacientes críticos, mesmo que o diagnostico de enfermagem da NANDA relate que os principais fatores de risco para o olho seco são observados no ambiente intensivo (Herdman; Kamitsuru, 2018). Nesse contexto, Fernandes et al. (2018), destaca que existem duas causas para o desenvolvimento do olho seco, como a deficiência na produção do filme lacrimal e o resulta em sintomas de desconforto, distúrbios visuais e instabilidade do filme lacrimal com danos potenciais à superfície ocular. Ademais, uma das complicações comuns em pacientes criticamente enfermos, resultantes da sedação ou coma, é o fechamento palpebral ineficaz. Logo, esses pacientes apresentam maior risco para o desenvolvimento de olho seco e, consequentemente, para a lesão de córnea (ARAÚJO et al., 2017). A atuação prática e as contribuições teóricas do enfermeiro sãoprimordiais diante da necessidade de um olhar amplo e humanizado quando se refere a um paciente sob cuidados de saúde, principalmente na assistência no ambiente intensivo. Logo, o enfermeiro possui um papel fundamental ao proporcionar um fluxo no atendimento de forma ordenada com ênfase no planejamento, na implementação de ações inerentes à profissão e, principalmente, como educador no qual poderá participar diretamente do processo de promoção e de qualidade de vida tanto da equipe sob sua responsabilidade ou para a reabilitação física e psíquica de um paciente (Oliveira et al., 2016).

Em adição, no que se refere aos principais fatores de risco para o desenvolvimento do olho seco em pacientes criticamente enfermos, a exposição do globo ocular, a sedação, a ventilação mecânica e a baixa temperatura da unidade de terapia intensiva, foram os principais fatores de risco relatados. Houve, ainda, relatos de que o cuidado de enfermagem realizado de forma inadequada é fator para o desenvolvimento de lesão na córnea. De forma a corroborar esses achados, segundo as literaturas disponíveis, dentre os fatores que podem levar ao surgimento do olho seco podemos destacar a presença de doenças neurológicas, pacientes sedados, em ventilação mecânica, submetidos a intubação e a oxigenioterapia, pacientes que fazem uso de sedativos, relaxantes musculares e drogas vasoativas, que apresentam escore na Escala de Coma de Glasgow (ECG) $<7$, o piscar de olhos inferior à 5 por minuto, que apresentam exposição do globo ocular, que apresentam um tempo de internação prolongada, edema periórbital, dentre outros (araújo et al., 2016; herdman; kamitsuru, 2018).

Entre os métodos preventivos para o olho seco, estudos clínicos inferem que o filme de polietileno que forma a câmara úmida é a opção mais eficaz, esse filme é um polímero transparente, flexível e não tóxico que tem função de evitar a evaporação de líquidos da cavidade ocular para o meio, dessa forma é mantida a umidade no local recoberto, formando a câmara úmida (KALHORI et al., 2016). Ademais, um estudo nacional, constatou que pacientes que utilizam o filme de polietileno apresentam risco $98 \%$ menor de desenvolver lesão na córnea, quando comparados aos pacientes que fazem uso de gaze embebecida em solução salina (werli-alvarenga, 2014). Todavia, o principal método de prevenção utilizado pelos enfermeiros em relação ao risco de olho seco, foi umidificação do globo ocular com a solução fisiológica a $0,09 \%$. procedimento que oferece graves riscos aos pacientes, pois estudos internacionais apontam que a aplicação de gaze com solução salina aumenta a prevalência e a gravidade de ceratopatia em pacientes críticos, portanto, seu uso não é

Quadro 1. Características dos Enfermeiros entrevistados, Belém, Pará, 2019

\begin{tabular}{|c|c|c|c|c|c|c|}
\hline Identificação & Estado Civil & Gênero & Idade & Pós-Graduação & Tempo de serviço no setor & O setor possui estrutura adequada \\
\hline E1 & União Estável & Feminino & 30 & Sim & 4 anos & Não \\
\hline E2 & Casada & Feminino & 49 & Sim & 1 ano & Sim \\
\hline E3 & Casado & Masculino & 34 & Sim & 6 anos & Sim \\
\hline E4 & Casada & Feminino & 37 & Sim & 8 anos & Sim \\
\hline E5 & Casado & Masculino & 45 & Sim & 12 anos & Sim \\
\hline E6 & Divorciado & Masculino & 39 & Sim & 7 anos & Sim \\
\hline E7 & União Estável & Feminino & 42 & Sim & 12 anos & Sim \\
\hline E8 & Solteiro & Masculino & 29 & Sim & 5 anos & Sim \\
\hline E9 & Divorciada & Feminino & 47 & Sim & 12 anos & Sim \\
\hline E10 & Casada & Feminino & 47 & Sim & 9 anos & Sim \\
\hline E11 & Casada & Feminino & 54 & Sim & 25 anos & Sim \\
\hline
\end{tabular}

Fonte: Pesquisa de campo, 2019

aumento da evaporação. No que concerne as unidades de terapia intensiva, o filme lacrimal está comprometido em virtude da desordem nos mecanismos responsáveis pela lubrificação e proteção ocular.A disfunção do filme lacrimal, conhecida como "olho seco", é uma alteração multifatorial das lágrimas e da superfície ocular que recomendado (davoodabady; rezaei; rezaei, 2018).outrossim, cada instituição apresenta variância de procedimentos de cuidados, porém, se tratando do olho seco as intervenções de cuidados podem ser agrupadas em três categorias: higiene ocular, prevenção da secura ocular e o fechamento ocular. a higiene ocular, está associada com 
métodos de limpeza, que podem incluir gazes ou cotonetes esterilizados, irrigados por solução salina ou água (OLIVEIRA et al., 2016). Somado a isso, a prevenção da secura ocular apresenta uma variedade de ações que podem ser desenvolvidas para manter ou promover o filme fisiológico de lágrimas e prevenir o ressecamento ocular, incluindo a câmera de umidade (filme polietileno), o colírio de metil-celulose, os lubrificantes, as lágrimas artificias, entre outros. Por sua vez, o fechamento ocular inclui o uso de adesivos, suturas, entre outros (Oliveira et al., 2016; herdman; kamitsuru, 2018). Por tanto, é imprescindível o conhecimento acerca das características clínicas dos pacientes com ressecamento ocular, pois possui uma estimada relevância, por permitir ao enfermeiro o diagnóstico correto, que possibilite planejamento adequado das ações de enfermagemdirecionadas à prevenção e ao controle de possíveis agravos à saúde ocular (Pimtombeira et al., 2018).Perante o exposto, consideramos ser imprescindível para os enfermeiros, a busca por novos conhecimentos para atender as necessidades de seus pacientes, promovendo, então, a mudança da assistência baseada na vivência diária por uma assistência baseada em evidências cientificas. Para isso, cursos de capacitação, atualização profissional, educação continuada e elaboração de protocolos são métodos imprescindíveis quando se almeja garantir assistência de alta qualidade aos pacientes com risco de olho seco.

\section{CONSIDERAÇÕES FINAIS}

A partir da análise dos resultados obtidos, compreendemos que os pontos de maior relevância sobre a temática foram abordados, assim como os objetivos da pesquisa foram alcançados, haja vista que os resultados permitiram reflexões sobre o entendimento dos enfermeiros intensivistas acerca do diagnóstico de enfermagem risco de olho seco, fatores de risco e os métodos e intervenções utilizadas durante as práticas cuidativas dos enfermeiros. Nesse caso, foi possível fazer comparações com os métodos disponíveis e recomendados pela literatura atual para a prevenção do risco de olho seco. No entanto, o conhecimento dos enfermeiros acerca da temática se mostrou fragilizado, ou seja, os resultados permitem inferir que o entendimento dos enfermeiros sobre a temática é superficial, pois não houve respostas consolidadas e detalhadas a respeito do conceito de risco do olho seco, bem como os principais fatores de risco discriminados nos diagnósticos de enfermagem da NANDA. Com isso, constatamos que a inclusão do diagnóstico de enfermagem risco de olho Seco na no processo de enfermagem da unidade é necessária e relevante, para a utilização nas atividades diárias do setor, uma vez que esse diagnóstico garante maior atenção e assistência com qualidade aos pacientes, assim como, o incentivo da educação continuada e a implantação de um protocolo padronizado para auxiliar no cuidado com o globo ocular, uma vez que a há grande rotatividade de profissionais dentro das unidades intensivas, dificultando a assistência contínua sem auxílio de um protocolo padrão.

\section{REFERÊNCIAS}

Araújo, D., D. Almeida, N., G. Silva, P., M., A. Ribeiro, N., S. WerliAlvarenga, A. andChianca, T., C., M. 2016. Predição de risco e incidência de olho seco em pacientes críticos. Revista LatinoAmericana de Enfermagem, 24:e2689.Acesso em: https://doi.org/10.1590/1518-8345.0897.2689.
Araújo, D., D. Werli-Alvarenga, A. Almeida, N., G. Silva, P., M., A. Macieira, T., G., R. andChianca, T., C., M. 2017. Concordância Inter avaliadores em avaliação da córnea de pacientes críticos. Revista de Enfermagem do Centro-Oeste Mineiro, 7:e1392. Acesso em: http://dx.doi.org/10.19175/recom.v7i0.1392.

Araújo, D., D. Ribeiro, N., S. andChianca, T., C., M. 2017. Efetividade do filme de polietileno na prevenção de olhos secos em pacientes críticos: revisão sistêmica. Revista Oficial do Conselho Federal de Enfermagem, 8:77-81. Acesso em: http://revista.cofen.gov.br/index.php/enfermagem/article/view/79 $0 / 357$.

Bardin, L. Análise de conteúdo. São Paulo: Edição 70. 2016.

Davoodabady, Z. Rezaei, K. and Rezaei, R. 2018. The Impact of Normal Saline on the Incidence of Exposure Keratopathy in Patients Hospitalized in Intensive Care Units. Iran J NursMidwifery Res., 23:57-60. Acesso em: https://doi.org/10.4103/ijnmr.IJNMR_187_16.

Fernandes, A., P., N., L. Araújo, J., N., M. Botarelli, F., R. Pitombeira, D., O. Ferreira Junior, M., A. Vitor, A., F. 2018. Dry Eye Syndrome in Intensive Care Units: a concept analysis. Revista Brasileira de Enfermagem [Internet], 71:1162-9. Acesso em: http://dx.doi.org/10.1590/0034-7167-2016-0582.

Herdman, T., H. Kamitsuru, S. Diagnósticos de enfermagem da NANDA: definições e classificação (2018-2020). 11. ed. Porto Alegre: Artmed, 2018

Kalhori, R., P. Ehsani, S. Daneshgar, F. Ashtarian, H. and Rezaei, M. 2016. Different nursing care methods for prevention of keratopathy among intensive care unit patients. Global Journal of Health Science, 8:4423-4434. Disponívelem: http://dx.doi.org/ $10.5539 /$ gjhs.v8n7p212.

Oliveira, J., B. Francalino, T., R. Silva, M., L., F. Araújo Júnior, A., C. Lima, L., R. 2016. Atuação do enfermeiro no controle de infecção hospitalar em unidade de terapia intensiva (UTI). Mostra Interdisciplinar do curso de Enfermagem, 2:2. Acesso em: http://publicacoesacademicas.unicatolicaquixada.edu.br/index.php $/$ mice/article/view/1143/919.

Pitombeira, D., O. Souza, A., M., L. Fernandes, A., P., N., L. Araújo, J., N., M. Silva, A., B. and Vitor, A., F. 2018. Características dos pacientes com ressecamento ocular internados em unidade de terapia intensiva. Revista Cogitare Enfermagem, 23:e. 53081. Acesso em: http://dx.doi.org/10.5380/ce.v23i2.53081.

Queiroz, A., G., S. Souza, L., P. Martelli, B., R. Lpihe, K., C. Benetti, H., A. Covrel, K., S. Siqueira, J., L. Feitosa, R., F. Pereira, J., T. 2018. O saber do enfermeiro no processo de diagnóstico e intervenção de enfermagem para risco de lesão na córnea. Revista Eletrônica Acervo Saúde, 14: Acesso em:http://dx.doi.org/ 10.25248/REAS325_2018.

Werli-Alvarenga, A. Efeito das intervenções de enfermagem na prevenção de lesão na córnea: ensaio clínico randomizado. 2014. 143 p. Tese (Doutorado em Enfermagem) - Universidade Federal de Minas Gerais, Escola de Enfermagem, Belo Horizonte, 2014.

Werli-Alvarenga, A. Ercole, F., F. Botoni, F., A. Oliveira, J., A., D., M., M., O. Chianca, T., C., M. 2016. Corneal injuries: incidence and risk factors in the Intensive Care Unit. Revista LatinoAmericana de Enfermagem, 19:1088-95. Acesso em: https://doi.org/10.1590/S0104-11692011000500005. 